\title{
PHOTOMETRIC PROPERTIES OF EXTRAGALACTIC BULGES
}

\author{
FRANCESCO BERTOLA \\ Department of Astronomy, University of Padova
}

\section{Introduction}

The "unresolved nuclear region", as Hubble (1926) indicated the bulge of spiral galaxies, became a physically distinct component with the introduction by Baade (1944) of the concept of stellar populations.

Since then, the role of the bulge itself and of its luminosity with respect to that of the disk is crucial in the context of understanding the Hubble sequence and the formation history of galaxies. For instance, it has been proposed to regard the Hubble sequence as a sequence of spheroidal luminosities (Meisels and Ostriker 1984) or as a sequence of decreasing bulge to disk ratios (van der Bergh 1976). Concerning the formation processes, in addition to the classical collapse picture (Eggen, Lynden-Bell, Sandage, 1963) where the bulge stars are formed before the formation of the disk, other possibilities are suggested. Bulges, with the exception of the very small ones characteristic of late-type spirals, could have been formed by merging of small objects (Larson 1990) or by two galaxies (Rix and White 1990). In any case bulges should have been formed before the disk, as indicated by their lower metallicity, so that, as stated by Binney and May (1986), disk galaxies probably started as undressed' spheroids.

It is therefore clear that the detailed study of bulges, both from the kinematic, photometric and chemical abundance point of view, will offer interesting clues on their role in galaxy formation.

\section{The bulge-disk decomposition}

The most direct information that can be derived from the photometric study of disk galaxies is the determination of the photometric parameters of the disk and bulge component, disregarding the presence of superimposed features such as bars, lenses, spiral arms and rings. Such parameters are the effective radius $r_{e}$ of the bulge and the corresponding surface brightness $\mu_{e}$, the ellipticity of the bulge isophotes, the central surface brightness of the disk and its scale length $\alpha$ and the ellipticity of the disk isophotes. The luminosity profile of the bulge is supposed to follow the $r^{1 / 4}$ law, while in the disk the surface brightness decreases exponentially. Additional information is represented by possible trends of the ellipticity and of the position angle of the major axis as a function of the distance from the center.

After the pioneering work of de Vaucouleurs (1959) several methods of decomposing the bulge and the disk component have been developed. They are reviewed by Simien (1989) and Capaccioli and Caon (1992). The modern techniques make use of the full two dimensional images obtained with CCD detectors and can be described as follows. The image is cleaned using masking techniques, removing disturbances such as spiral arms, dust lanes and superimposed star images. Then a 
smooth model of the disk is constructed using the parameters derived in the outer parts of the image and subtracted from the image of the galaxy. What remains is the bulge component, from which a smooth model is also constructed. The model galaxy, obtained by adding the two components, is then compared with the original. This procedure is repeated several times adjusting the input parameters of the models until the residuals (galaxy minus model galaxy) are minimized.

The bulge to disk ratio (B/D), namely the ratio between the luminosity of the disk and that of the bulge, derived with the above methods is an important parameter characterizing disk galaxies .

One of the most interesting points is to establish how the $\mathrm{B} / \mathrm{D}$ ratio is correlated with the morphological type, as suggested by the original Hubble criteria of classification, and, if this correlation is present, which are the separated roles of the disk and the bulge.

In the morphological approach of van der Bergh (1976) $S 0$ galaxies are considered as forming a sequence of decreasing B/D parallel to that of spirals, thus they are removed from being a transition between ellipticals and spirals. This point has been contradicted by the photometric decomposition of Simien and de Vaucouleurs (1986) whe find a correlation between $B / D$ and the morphological type, B/D being highest for $S 0$ galaxies. The decreasing $\mathrm{B} / \mathrm{D}$ for late-type galaxies is mainly due to a decreasing luminosity of the bulge. Similar results were also found by Kent (1985). However the analysis of a large sample of galaxies led Kodaira et al (1986) to the result that the mean absolute magnitude of disks and bulges is nearly constant over the entire sample of morphological types. They concluded that the Hubble type does not show a tight correlation with either the spheroid magnitude, the disk magnitude or the $B / D$ ratio.

The analysis of a sample of 35 spirals and $19 S 0$ galaxies in the Virgo Cluster (Bertola and D'Onofrio 1992) shows a slight increase of the absolute magnitude of the disk while the luminosity of the bulge decreases from $S 0$ to $S c$ type. In this way the $\mathrm{D} / \mathrm{B}$ ratio varies from 0.5 to 2 , a much smaller range that the one given by Simien and de Vaucouleurs (1986) (Fig.1). These discrepancies could be due to errors in the decomposition, different classification criteria and incompleteness of the samples. Further studies should clarify the point.

\section{The detection of hidden disks}

One of the major results of the photometric studies of early type galaxies in recent years has been the discovery of faint disks relative to the spheroidal component and therefore almost hidden in it. Galaxies with such disks were previously classified as ellipticals, they are often called "disky" ellipticals in contrast with "boxy" ellipticals, where no trace of disk is present and at the same time the outer isophotes show departures from the ellipticity towards squared shapes.

The hidden disks are usually found by representing the departures from ellipticity of the isophotes by means of a Fourier analysis. If the coefficient $a_{4}$ is positive, it implies a pointed structure of the isophotes, indicating the presence of the disk. This method has been extensively used by Bender et al $(1988,1989)$, who established that disky and boxy ellipticals are characterized by different physical properties. 


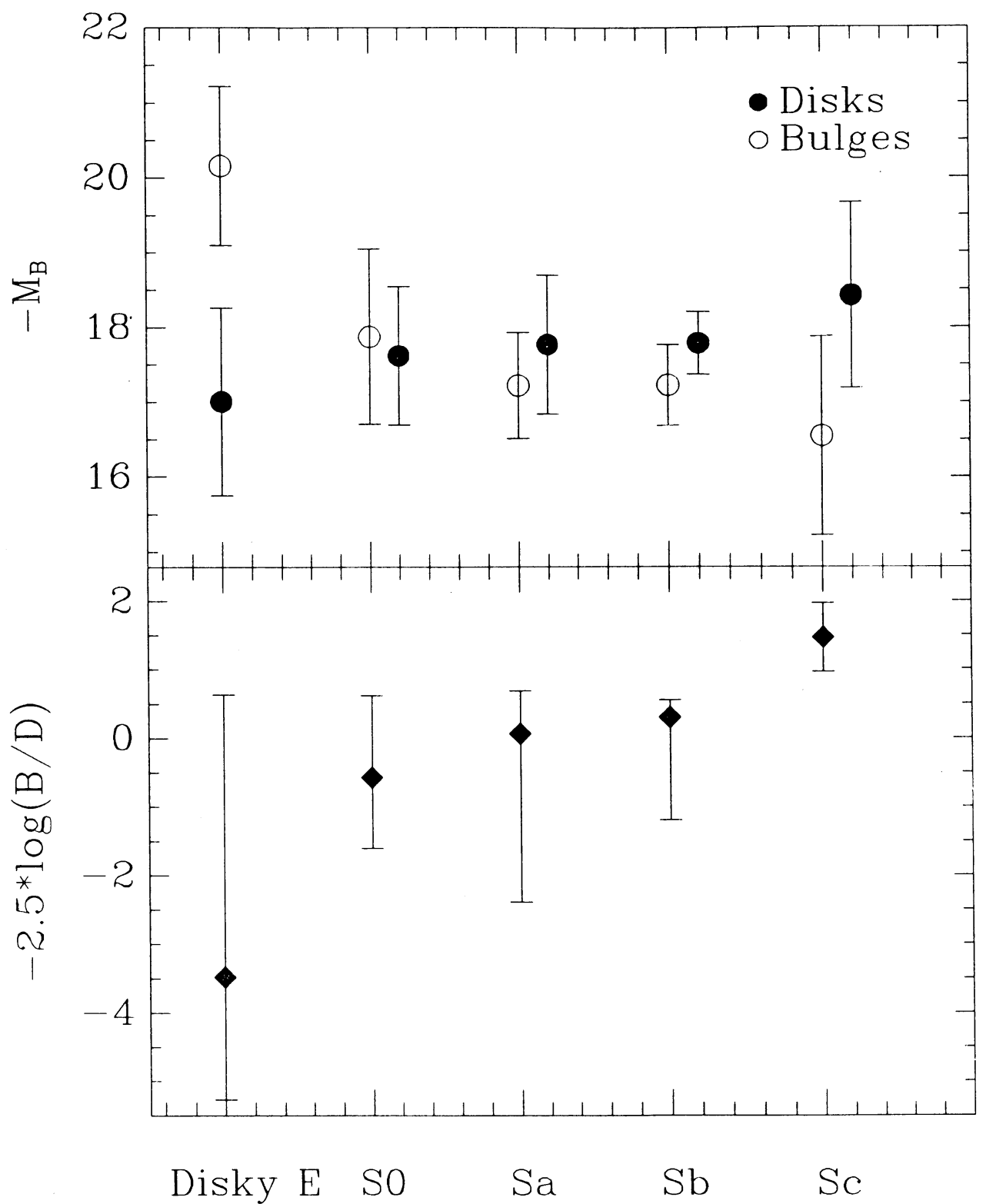

Fig. 1. Absolute magnitudes of disks and bulges and B/D ratios for a sample of $S 0$ and spiral galaxies in the Virgo Cluster (Bertola and D'Onofrio 1992). The available data on "disky" ellipticals are also plotted. 
A positive $a_{4}$ coefficient indicates the presence of a disk but not its parameters. In order to derive the photometric properties of the disk it is necessary to decompose the bulge and disk in a way similar to that described above. However the input parameters necessary to construct the model of the disk to be subtracted are not so easy to select. Scorza and Bender (1990) in decomposing the galaxy NGC 3610 selected the input parameters in such a way that the isophotes of the bulge would appear perfectly elliptical after the subtraction of an exponential disk. Also, after subtraction of the disk component the deviation from the $r^{1 / 4}$ law on the luminosity profile of the bulge are largely reduced. In this way it has been possible to find disks whose consistence is between $15 \%$ to $2 \%$ of the luminosity of the bulge. Simien and Michard (1990) developed a similar method, which does not imply the assumption that the disk is exponential.

Rix and White (1990) have studied the detectability of the hidden disks in elliptical galaxies, which is a function of the $\mathrm{D} / \mathrm{B}$ ratio and of the viewing angles. Face on disks are generally more difficult to detect than edge on ones and their photometric signatures reside mainly on the deviations from the $r^{1 / 4}$ law. On the contrary edge on disks produce noticeably positive values of the $a_{4}$ parameter. Rix and White estimate that $50 \%$ of all disks with a $\mathrm{D} / \mathrm{B}<0.25$ can not be detected by photometric means. Disks in elliptical galaxies can be detected also spectroscopically by studying the stellar line profiles, as they are produced by the two components. Rix and White (1992) were able to derive in this way the luminosity profiles of the disk and the bulge. However, the most appropriate way to decompose disk and bulge is to use both photometric and kinematical data. This method has been successfully applied by Cinzano and van der Marel (1992) to NGC 2974. They were not obliged to make assumptions on the perfect ellipticity of the bulge isophotes due to the presence of the kinematical constraints. In Fig. 1 mean absolute magnitudes of the disk and the bulge and the mean $\mathrm{B} / \mathrm{D}$ ratio are plotted also for a sample of seven "disky" ellipticals present in the literature (Scorza and Bender 1990; Capaccioli et al. 1991; Vader and Vigroux 1991; Scorza and Bender 1992; Cinzano and van der Marel 1992). The mean $D / B$ ratio of 0.04 is produced by a slightly lower luminosity of the disk than in $S 0$ and spiral galaxies and by a much higher luminosity of the bulge.

\section{The bulges and the fundamental plane}

It is well known that in the three dimensional space defined by the two photometric parameters $\mu_{e}$ and $r_{e}$ and the central velocity dispersion, elliptical galaxies show a strong tendency to be concentrated in a plane called the "fundamental plane". In a recent discussion of the structural properties of dynamically hot galaxies Bender, Burstein and Faber (1992) included, in addition to ellipticals, bulges of disk galaxies. They find that bulges share common properties with ellipticals of intermediate luminosity $\left(-20.5<M_{B}<-18.5\right)$, both looking edge-on and face-on at the fundamental plane. There is a tendency for the representative points of the bulges to lie slightly below the plane, but it is not clear whether this might be a genuinely lower $\mathrm{M} / \mathrm{L}$ than in ellipticals. In the edge on view of the fundamental plane bulges and ellipticals of intermediate luminosity occupy the central position while at the two 
- Bulges of $\mathrm{Sb} / \mathrm{Sc}$

- BCG's

$\triangle$ Dwarfs
Ellipticals

*alaxies hosting a QSO

- Bulges of SO/Sa

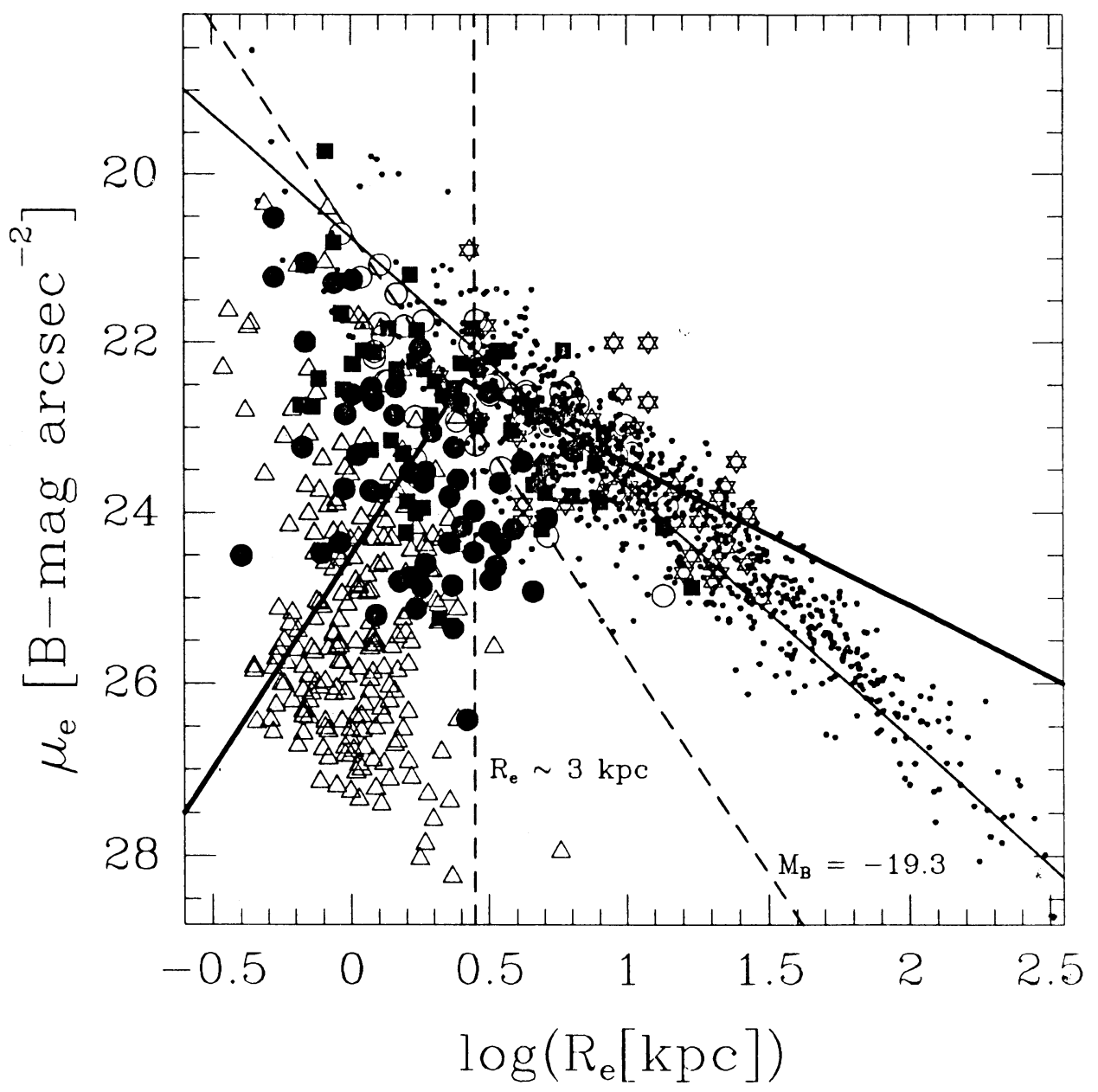

Fig. 2. Elliptical galaxies and bulges in the $\mu_{e}, R e$ plane according to Capaccioli et al. (1992). The thick line represents the relationship by Binggelli et al. (1984). 
extreme are faint ellipticals on one side and dwarf ellipticals on the other. According to Bender et al. (1992) the discriminating property is the degree of anisotropy in the velocity distribution. Bulges and intermediate ellipticals are associated since they both are isotropic rotators (Davies et al. 1983). Since giant ellipticals tend to be "boxy" and intermediate luminosity ellipticals tend to be "disky", it is suggested that the distinction between these two kinds of galaxies is due to the absence or presence of a disk. Such a disk is not recognizable in a visual inspection of the ellipticals of intermediate luminosity, characterized by an higher bulge to disk ratio than ordinary $S 0$ 's and spirals to which they are related. The same mixing of intermediate luminosity ellipticals and bulges is present also when the fundamental plane is projected into the plane defined by the two photometric parameters $\mu_{e}, r_{e}$ (Fig.2) (Capaccioli et al. 1992) with a tendency of disky ellipticals to concentrate toward the high luminosity lines.

Recent numerical simulations seem to cast some doubts on the interpretation that disky isophotes generally indicates the presence of a stellar disk. By studying elliptical galaxies as the result of a dissipationless collapse Stiavelli et al. (1991) found that the isophotal shape varies with the viewing angles, giving to the same galaxies either the "disky" or the "boxy" appearance. Similar results have been obtained by Governato et al. (1992), who investigated the properties of merger remnants from two spherical galaxies and from a disk and a spherical galaxy. The isophotes in the first case tend to be "boxy" while in the second case there is a marked tendency to be "disky". However they are unable to reproduce objects with high degrees of diskyness, as are often observed.

\section{The box- and peanut-shaped bulges}

$S 0$ and spiral galaxies, when seen on edge, often reveal bulges whose shape is box like or even peanut like, namely with a depression of the isophotes along the minor axis. This phenomenon has been known since long time and lists of galaxies exhibiting such peculiar bulges were published by Jarvis (1986), Shaw (1987) and Souza and dos Anjos (1987). Shaw (1987) finds that $20 \%$ of disk galaxies exhibit a box or peanut-shaped bulge. This percentage could be even higher taking into account that the peculiarity is not easily recognizable in late-type spirals. According to de Souza and dos Anjos (1987) this frequency, at least for $S 0$ galaxies, is consistent with the idea that all of them are barred galaxies as invoked by some recent numerical simulations. Jarvis (1986) has studied the occurence of box and peanut-shaped bulges in galaxies of different Hubble type, concluding that there is a strong tendency for them to occur in $S 0$ and $S a b$ to $S b$ types. He also finds that $63 \%$ have peanut-shaped bulges, compared to box-shaped ones.

Shaw (1992) has recently estimated that the extra light, producing the box/peanut shape, after the subtraction of a model bulge, is $5-15 \%$ of that in the bulge as a whole. This luminosity excess appears greater in later type galaxies, where the bulges are less prominent. Among the different mechanisms proposed to explain the formation of box/peanut bulges, such as external torques (May et al. 1985), merging (Binney and Petron 1985) and the presence of a bar (Combes et al. 1990), the latter seems to be the most promising one. By means of three-dimension N-body 


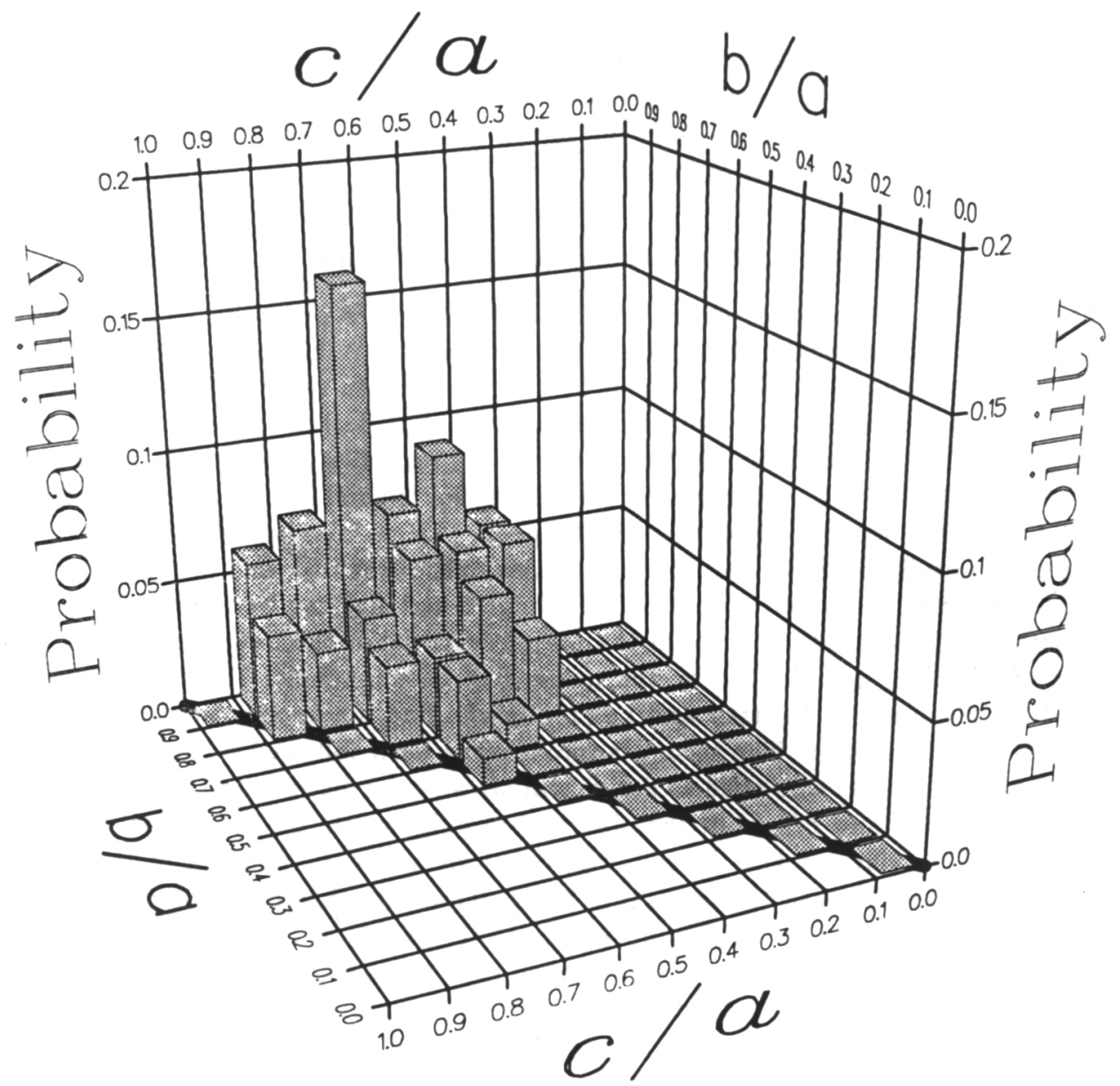

Fig. 3. The distribution function of the intrinsic axial ratios of bulges derived by Bertola et al. (1991) on the assumption that disks are circular. 
simulations Combes et al. (1990) find that bars develop a structure, which is seen superimposed on the spheroidal bulge, and gives the peanut or boxy appearance to the bulge according to the viewing angle.

There is a general consensus of the fact that boxy shapes in ellipticals are of a different nature than those of bulges.

\section{The intrinsic shape of bulges}

The fact that the intrinsic shape of elliptical galaxies is generally triaxial is supported by the observation of the isophotal twisting (e.g. Williams and Schwarzschild 1979, Bertola and Galletta 1979) and by the presence, in several cases, of a stellar velocity gradient along the projected minor axis (e.g. Bertola et al. 1989, Franx et al. 1989). Due to the similarity between several properties of ellipticals and bulges one is tempted to ask whether they also share the triaxial shape. Indeed Lindblad (1956) was the first to argue that the bulge of M31 is triaxial on the basis of the observed misalignment of the isophotal major axis of the disk and of the bulge, not expected if both components are oblate. Bulge triaxiality can be indicated both by variations of the position angle of the major axis within the bulge itself, as in the case of elliptical galaxies, or by the bulge disk misalignment. The first approach has been followed by Zaritsky and Le (1986) who find major axis twisting in all 11 bulges of their sample. The second approach has been followed by Bertola, Vietri and Zeilinger (1991) who tried to derive statistically the distribution function of the axial ratios $b / a$ and $c / a$ from the observed misalignments on the hypothesis that the disks are axisymmetric. In this way it has been found that half of the bulges are close to oblate, with the remaining being definitely triaxial (Fig.3). However, a subsequent investigation by Fasano et al. (1992) bäsed on the distribution of the apparent axial ratios of disks has revealed that the assumption of axisymmetry is not correct. In fact broad intrinsic distribution functions peaked at $b / a$ in the range $0.8 \div 0.9$ for both $S 0$ s and spirals were derived (Fig.4). Similar results were also found by Huizinga and van Albada (1992) and by Franx and de Zeeuw (1992). The latter authors, however, point out that these ellipticities are too large when considering the small scatter present in the Tully-Fisher relation.

The fact that disks are triaxial modifies the distribution of Fig.3, in the sense that bulges tend to be more triaxial if they are elongated along the same direction as the disk, while they are more oblate if the two directions are perpendicular.

\section{Concluding remarks}

The photometric studies of the bulges pose several questions. Here are a few:

i) Do $S 0$ galaxies represent a transitional stage in the Hubble classification scheme or do they form a sequence parallel to that of spirals in terms of B/D ratio ? According to Simien and de Vaucouleurs (1986) they represent a transitional stage. However the data of Fig. 1 suggest that they do not behave differently from $S a$ and $S b$ galaxies.

ii) Do disky ellipticals constitute the natural continuation of the $S 0$ s and spirals ? According to the study of their properties in the fundamental plane Bender 


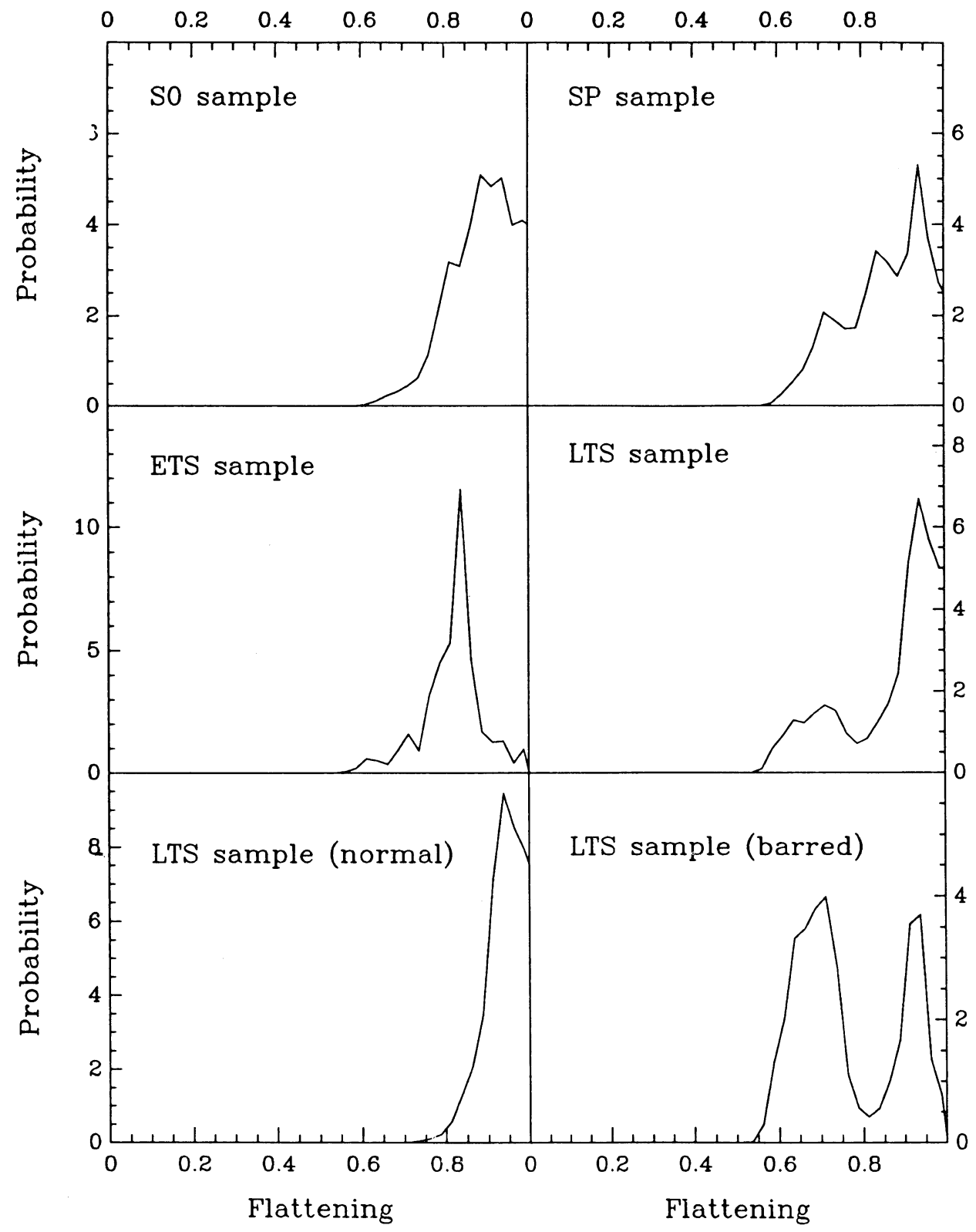

Fig. 4. The flattening distribution functions for disks derived by Fasano et al. (1992) for a sample of $S 0$ and spirals galaxies (top panels). The spirals are subdivided into early-type and late-type in the middle panels. Late-type spirals are subdivided into normal and barred in the bottom panels. Notice the effect of the bar. 
et al. (1992) conclude that bulges and ellipticals of intermediate luminosity belong to one smooth, unbroken sequence. However, according to the still scarce data values of Fig. 1 there seems to be a discontinuity in the values of the $B / D$ ratio.

iii) Is there a dichotomy between "disky" and "boxy" ellipticals or do they belong to the same sequence? Considering the tendency of the disk to become weaker in disky ellipticals, even weaker disks in galaxies with bulges of much higher luminosity would become undetectable.

iii) Are all bulges rotationally flattened ? This conclusion has been reached almost ten years ago by Davies et al. (1983). However, we now possess both dynamical evidence (Bertola et al. 1990; Gerhard et al. 1990) and photometric evidence (Bertola et al. 1991) that a fraction of bulges are triaxial. This suggests the presence of a degree of velocity anisotropy, which would be worth to investigating.

\section{Acknowledgements}

It is a pleasure to thank P. Amico, C. Bender, M. Capaccioli, P. Cinzano, M. D'Onofrio, A. Pizzella and C. Scorza. for useful discussion. J. Pesce was so kind to read the manuscript.

\section{References}

Baade, W.: 1944, Ap. J. 100, 137

Bender, R., Burstein, D., Faber, S.M.: 1992, Ap. J. in press

Bender, R., Döbereiner, S., Möllenhoff, C.: 1988, Astron. \& Astrophys. suppl. ser. 74, 385

Bender, R., Surma, P., Döbereiner, S., Möllenhoff, C., Madejsky, R.: 1989, Astron. \& Astrophys. 217, 35

Bertola, F., Capaccioli, M., Galletta, G., Rampazzo, R.: 1989, Astron. \& Astrophy. 192, 24

Bertola, F., D'Onofrio M.: 1992, in preparation ,

Bertola, F., Galletta, G.: 1979, Astron. \& Astrophys. 77, 363

Bertola, F., Rubin, V.C., Zeilinger, W.W.: 1989, Ap. J. Letters 345, L29

Bertola, F., Vietri, M., Zeilinger, W.W.: 1991, Ap. J. 374, L13

Binggelli, B., Sandage, A., Tarenghi, M:: 1984, Astron. J. 89, 64

Binney, J., May, A.: 1986, M.N.R.A.S. 218,743

Binney, J., Petrou, M.: 1985, M.N.R.A.S 214, 449

Capaccioli, M., Caon, N.: 1992, Morphological and Physical Classification of galaxies, Longo G., Capaccioli M., Busarello G.: Kluwer Academic.

Capaccioli, M., Caon, N., D'Onofrio, M.: 1992, M.N.R.A.S in press

Capaccioli, M., Vietri, M., Held, E., Lorenz, H.: 1991, Ap. J. 371, 535

Carter, D.: 1987, Ap. J. 312, 514

Cinzano, P., van der Marel, R.P.: 1992, Structure, Dynamics and chemical evolution of early-type galaxies., J. Danziger: ESO Garching

Combes, F., Debbash, F., Friedli, D., Pfenniger, D.: 1990, Astron. \& Astrophys. 233, 82

Davies, R.L., Efstathion, G., Fall, S.M., Illingworth, G.D., Schechter, P.: 1983, Ap.J. 266, 41

de Souza, R.E., dos Anjos: 1987, Astron. \& Astrophys. suppl. 70, 465

de Vaucouleurs, G.: 1958, Ap. J. 128, 465

Eggen, O.J., Lunden-Bell, D., Sandage, A.R.: 1963, Ap. J. 136, 748

Fasano, M., Amico, P., Bertola, F., Vio, R., Zeilinger, W.W.: 1992, M.N.R.A.S. in press

Franx, M., de Zeeuw, P.T.: 1992, Ap. J. in press

Franx, M., Illingworth, G., Heckman, T.: 1989, Ap. J. 344, 613

Governato, F., Reduzzi, L., Rampazzo, R.: 1992, M.N.R.A.S in press

Huizinga, J.E., van Albada, T.S.: 1992, M.N.R.A.S in press 
Hubble, E.P.: 1926, Ap. J. 64, 321

Jarvis, B.J.: 1986, A.J. 81, 65

Kent, S.M.: 1985, Ap. J. Suppl. 58, 115

Kodaira, K., Watenabe, M., Okamura, S.: 1986, Ap. J. Suppl. 62, 703

Lindblad, B.: 1956, Stockholm Obs. Ann. Vol.19,N.2

May, A., van Albada, T.S., Norman, C.A.: 1985, M.N.R.A.S. 214, 131

Meisels, A., Ostriker, J.P.: 1984, A. J. 89, 1451

Rix, H.-W., White, D.M.: 1990, Ap. J. 362, 52

Rix, H.-W., White, D.M.: 1992, M.N.R.A.S 254, 389

Scorza, C., Bender, R.: 1990, Astron. \& Astrophys. 235, 49

Scorza, C., Bender, R.: 1992, Morphological and Physical Classification of galaxies, Longo G., Capaccioli M., Busarello G.: Kluwer Academies, 389

Shaw, M.A.: 1987, M.N.R.A.S 229, 691

Shaw, M.A.: 1992 M.N.R.A.S in press

Simien, F.: 1989, The World of Galaxies, Corwin H.G. and Bottinelli L.: Springer Verlag, 293

Simien, F., de Vaucouleurs, G.: 1986, Ap. J. 302, 564

Stiavelli, M., Londrillo, P., Messina, A.: 1991, M.N.R.A.S 251, 57p

van den Bergh, S.: 1976, Ap. J. 206, 883

Williams, T.B., Schwarzschild, M.: 1979, Ap. J. 227, 56

Zaritsky, D., Lo, K.Y.: 1986, Ap. J. 303, 56

\section{DISCUSSION}

Sellwood: I was delighted to hear that the fraction of box shaped bulges is slowly rising as observers look more carefully. But I must caution about pointing to the coincidence of $30 \%$ fraction of box bulges, compared to $30 \%$ of galaxies that are barred. Because when we see a galaxy edge on, the bar can be aligned at any random angle to the line of sight. Therefore we would expect the fraction of box bulges, if they were made from bars, to be less than the fraction of bars. We should also check: whether the dimensions of these two things are comparable, before we really start being confident that this is an explanation for the peanut phenomenon.

Rix: I have a comment on your analysis of the shape of the disks. It seems you find that the disks in late type spirals and barred systems, deviate more strongly from axisymmetric. Both of those systems, more or less by definition for the late type spirals and for bars, are strong two armed spirals. Also, you inferred the triaxiality from the lack of systems with unit axis ratio. Is it clear to you in those cases that the axis ratios in those case do not only reflect the spiral arms, but have something to do with the underlying disk?

Bertola: We say no at this point and as I said, we took the data from the literature and also a sample of 100 galaxies, which we analyzed. We tried to analyze the influence of the spiral arms. What we found was that the disk is dominant and there is not so much an effect from the spiral arms. Of course in the inner regions this would not be the case and they would cause a twisting of the isophotes. 


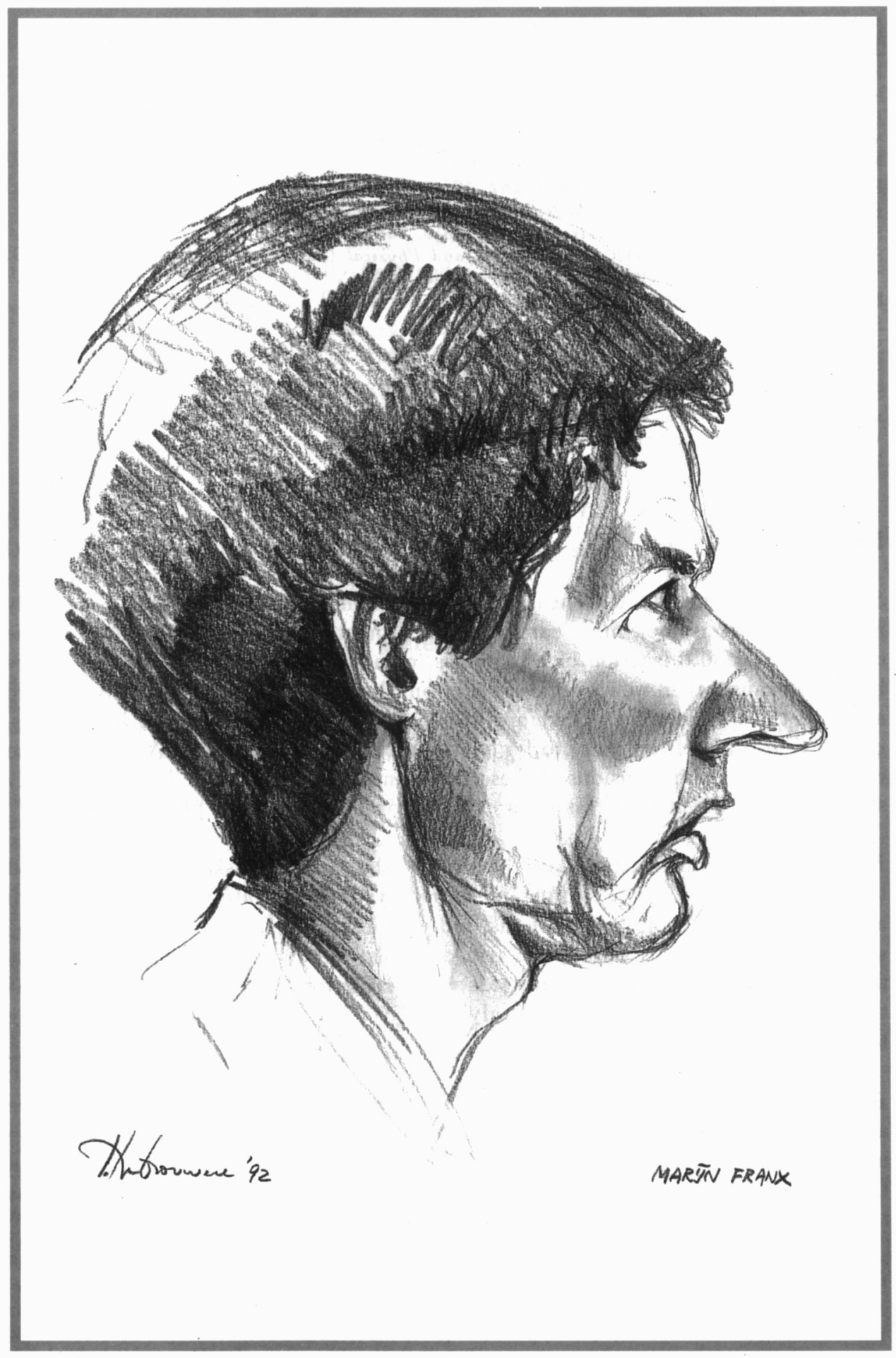

